\title{
Analysis on the Function Evaluation of One Stage Anterior Internal Fixation for the Treatment of the Cervical Vertebra and Neck Segmental Spinal
}

\author{
Beilei Zhan ${ }^{1, a}$, Dong Chen ${ }^{1,{ }^{*}}$, Zhou Ye ${ }^{1}$, Yunzhong Zhan ${ }^{1}$, Jun Qian ${ }^{1}$ \\ ${ }^{1}$ Department of Orthopedics, Quzhou People's Hospital, Quzhou, 324000, China \\ azhanzhan@126.com
}

*Corresponding author yezhou@163.com

\begin{abstract}
Keywords: Issue before; Internal fixation; Cervical spine; Cervical segmental spinal
\end{abstract}
\begin{abstract}
Objective: To observation and analysis one stage anterior internal fixation treatment of cervical vertebra and neck thoracic segment spine and its clinical efficacy. Methods: We select 10 patients with cervical and thoracic segments neck spine line issue of anterior cervical lesions cleared and iliac bone graft titanium plate fixation from March 2013 to August 2014. The anti-tb treatment is $>9$ months after surgery. Results: surgical blood loss was $325 \mathrm{ml} \pm 25 \mathrm{ml}$. 10 cases received following-up, following-up time was $10 \sim 12$ months, following-up period tuberculosis recurrence and internal fixation failure was not found. The spinal cord and nerve root compression symptoms improved to some extent, JOA score is increased from $10.8 \pm 1.1$ points preoperatively to $15.4 \pm 0.9$ points after the operation. Conclusion: One stage anterior internal fixation treatment of cervical vertebra and early cervical segmental spinal can obtain satisfactory curative effect. Thoroughly remove lesions $360^{\circ}$, the treatment that jointed before and after internal fixation and bone graft system of anti-tuberculosis treatment is the key to obtain satisfactory curative effect.
\end{abstract}

\section{Introduction}

The cervical and thoracic posterior equipment fixed on the changed dramatically. Although traditional wire between the spinouts process of fixed and relatively less use of vertebral plate steel wire fixation method still has good clinical application prospects, but often need more strong fixation devices, especially for the cases of cervical instability significantly. Degenerative factors cause nerve compression of cases often requires decompression, such as vertebral lamina resection, which makes the vast majority of steel wire fixed way cannot be used. The traumatic deformities often needs to be stronger and fixed to support the unstable after anterior column or columns. Cervical lateral mass screws under technology as well as the recent application of cervical and upper thoracic pedicle screw system has been widely recognition, has produced a new generation can be applied to even the most complicated cervical thoracic deformity reconstruction plants within the system. Selection in March 2013 to August 2014 in 10 patients with cervical and thoracic segments neck spine line issue of anterior cervical lesions cleared and iliac bone graft titanium plate fixation, now report as follows.

\section{Materials and Methods}

\section{Materials}

We select 10 patients with cervical and thoracic segments neck spine line issue of anterior cervical lesions cleared and iliac bone graft titanium plate fixation from March 2013 to August 2014. 6 cases are male, 4 cases are female; At age is 27 to 45 years old. Lesions involving the segment are C6 T1. 10 cases are characterized by the neck dull pain, fatigue or rotate after the flexion and extension activities pain intensified, can reduce after bed rest. With different degree of spinal cord and nerve root compression symptoms, chest band 4 cases. Preoperative JOA score was $10.8 \pm 1.1$ points. Preoperative blood sedimentation is $25 \sim 95 \mathrm{~mm} / \mathrm{h}$. 


\section{Methods}

1) The complete resection of the tumor and bone graft choice steel plate, according to the degree of cervical, with curved wrench to change the standard of steel plate bending, use a wrench firmly grasp the steel plate and the pressure to the vertebral body. 2) to drill into the appropriate set of drill sets, drill sets and is consistent with wrench, can accurately adjust the direction of the drill bit, and can protect the surrounding soft tissue. Pay attention to the upper and lower end screw hole to the Angle of the head and tail; choose due to a depth of $13 \mathrm{~mm}$ drill bit. After drilling tapping, with the appropriate tap is by bit coat line fully tapping. Screw 3) through a wrench and screw down the screwing in borehole, lest hinder the board the operating nut position was too high. The second screw should be fixed in the first diagonal screw position, other screw fixed in turn. When the four terminal screws are fixed, to remove a plate, will be fully tighten the screw. 4) When necessary by steel plate fixed screw holes in the middle of the piece of bone graft. Screw position and Angle can be adjusted according to the need of the performer appropriately, but must pay attention to the important structure around don't hurt.

\section{Results}

The surgical blood loss for $325 \mathrm{ml} \pm 25 \mathrm{ml} .5$ cases received follow-up, follow-up time was $10 \sim$ 12 months, follow-up period tuberculosis recurrence and internal fixation failure was not found. The spinal cord and nerve root compression symptoms improved to some extent, JOA score increased from $10.8 \pm 1.1$ points preoperatively to $15.4 \pm 0.9$ points after the operation. Show in Fig. 1 and Table 1.

Table 1: Compared JOA score before and after treatment

\begin{tabular}{lll}
\hline Group & Number & JOA score \\
\hline $\begin{array}{l}\text { Before } \\
\text { treatment }\end{array}$ & 10 & $10.8 \pm 1.1$ \\
$\begin{array}{l}\text { After } \\
\text { treatment }\end{array}$ & 10 & $15.4 \pm 0.9$ \\
$\begin{array}{l}2 \\
P\end{array}$ & & 10.967 \\
& & $<0.05$ \\
\hline
\end{tabular}

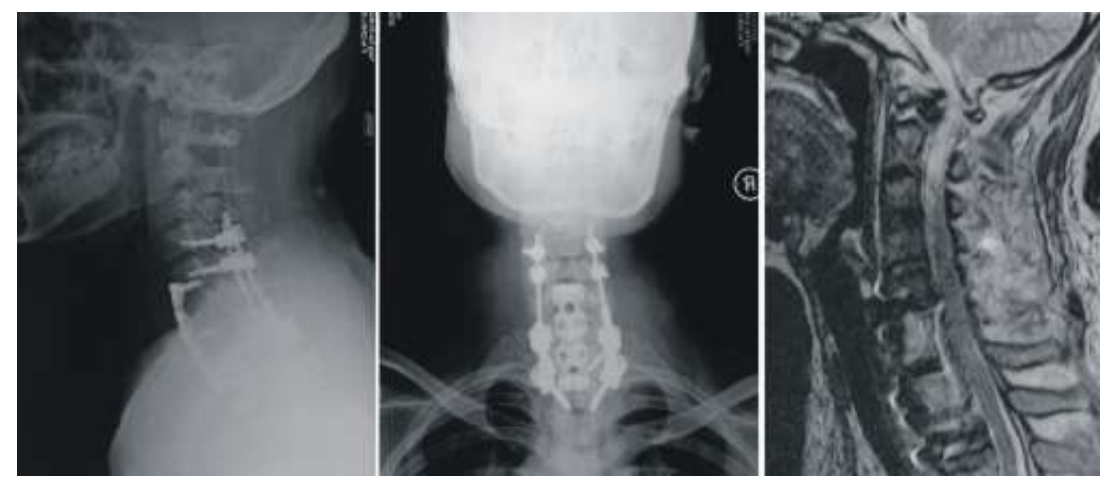

Fig 1 One Stage Anterior Internal Fixation for the Treatment of the Cervical Vertebra and Neck Segmental Spinal

\section{Discussion}

To tuberculosis kitchen local people have long into the curative effect of internal fixation device, and conservative attitude, but both basic and clinical research suggests that a fixed term will not 
increase the risk of infection and the spread of TB, clinical effect is better. About in the choice of internal fixation, most scholars advocate posterior fixation, direct anterior fixation when plants located in the lesions of local, have caused TB continues to not more, such as infection spread dangerous, but the posterior fixation prior incision, and have more bleeding, shortcomings and so on large trauma, surgical time. Ming Jianzhang reported under the preoperative preparation such as anterior fixation is safe, in terms of deformity correction, spinal stability maintenance the curative effect of the posterior fixation is better.

Cervical thoracic posterior stability of the border section there are a lot of indication. Vertebral body subtotal resection or disc $\mathrm{C} 6 \sim \mathrm{T} 1$ after anterior formation can be simply treated with posterior fusion, can often be found that this level of posterior fixation and fusion will eventually lead to the front. Trauma, such as chest neck border small joint fracture and dislocation, often can use simple posterior surgery to treat, can be open reduction or closed reduction. Myelopathic type was the expansion of cervical spondylosis anterior decompression often need to strengthen, posterior fixation and if the relief involving the cervical spine, the posterior fixation often need to border neck thoracic segment. Ankylosing spondylitis patients the posterior cervical spine deformity of the bone is usually conducted in C6 $\sim \mathrm{T} 1$ segment, although this kind of surgery is not fixed, and with posterior fixation can improve the convergence rate, and in some cases of selective can save head ring bracket vest. Finally, the reconstruction of the lamina resection after cervical vertebra protrusion deformity after often need to anterior and posterior surgery at the same time, due to the operation range is larger, so at the same time, posterior fixation methods often used before, often reaching the thoracic posterior fixation.

Neck thoracic segment between the posterior fixations without absolutely contraindicated, however preoperative diagnosis and surgical approach, different choice, made using the fixed type. As already mentioned the influence of the anatomical structure makes the choice of internal fixation by certain restrictions. Resection of the cervical vertebral lamina decompression will not be any wire fixation between the processes, unless the increase the fusion segment can adopt. Although the small joints in steel wire fixed after decompression is an option, but the intensity of a larger plate and rod system can provide better stability. Epidural abscess is a direct line surgery fixed relatively contraindicated. Many performer for fear of an acute infection and avoid using posterior fixation. But after arrival if the relief is not stable, the posterior fixation surgery can wait after acute infection control can be performed.

The advantages of this procedure is to overcome the multiple segmental cervical thoracic segment of spinal tuberculosis, anterior plate fixation difficult problems fixed reliable, the operation risk is smaller than the road ahead, at the same time can effectively remove lesions, bone graft. The current clinical mainly uses the anterior lesion clearance and bone graft fusion and internal fixation of the surgical treatment of cervical segmental spinal tuberculosis. Anterior surgery can effectively remove lesions and bone graft, but as a result of the segmental physiological radian characteristic, at present all internal fixation materials for the integration of a cervical thoracic segment fixed all difficulties. If such as the typical cases, anterior internal fixation, the screws should be fixed on the C6 and T3 vertebral bodies can be stable, but it's hard to anterior plate across such a long segment. Additional T3 vertebral bodies near the aortic arch, deep location, place the plate will increase the risk of surgery. We choose posterior pedicle screw fixation, solved the problem of the anterior plate fixation difficult, and reduces the risk of surgery. The group of eight cases, combined anterior and posterior approach was used for treatment, has obtained the good curative effect, which this is a reasonable mode of operation. The drawback is that requires two mammoplasty incision, to some extent, increased the surgical trauma and time.

Border neck thoracic posterior fixation usually requires the patient in the prone position, one of the few cases also can use the seat, need special when head bolt of danger at this time. Be careful especially when the mercy to the patient, especially the sides of the face, eyes must be without pressure, because of long time compression can cause unilateral or bilateral loss of vision. Although the horseshoe shape of the head is a choice, but must also mat is very good, and the anesthesiologist, often should check the eyes, to ensure that no head rotation and cause eye pressure side or both 
sides. In order to significantly reduce the risk of this position make the eye pressure, the patient can be placed in Mayfield head frame. Method is three needles on the head, then turned to 40-60 lb, Mayfield heads and surgical bed multidirectional joints between the performer is a good way to adjust the position of the head and neck. After adjusting the location of the patient's head and neck, must consider the patient's specific surgical indication. Cervical vertebra tube significant patients with myelopathic type cervical vertebra disease and don't make the neck with a, because it can increase the compression of the spinal cord, cause nerve damage and even paralysis occurs before the surgery. If caused by fracture or tumor is not stable, so the patients from the hypothesis to be especially careful when prone position, and then make the head and neck in a natural position when the head frame lock. If there is worry about adjusting position may cause damage to the spinal cord, you can use the spinal cord. Baseline signal can be when patients were determined in the transport of bed, position adjust to determine again later, in order to ensure that the baseline signal without change. Usually on both sides of the arm is put in the trunk, such as excessive Yang, will influence the performer's stance and affect perspective, even if the arm on both sides and use tape to tie up, pull the shoulder, on X-ray plain film to observe the bony structure of the neck chest junction is still very difficult. Once in a while when the patient is relatively small, the side of the border neck chest images can be easily collected.

Reconstruction of the cervical posterior internal fixation system, according to the principle can be divided into steel wire (titanium cable) fixed, fixed, lateral mass fixation and vertebral powder pedicle fixation. Because of cervical vertebra and neck thoracic tumor patients with cervical more after the destruction of the column structure, steel wire and vertebral powder after this dependence column complete can't adapt to the need, and the lateral mass fixation and pedicle fixation is not affected by structural integrity after cervical vertebra, good mechanical strength, fixed segmental short, postoperative nursing is convenient, thus has the use of a broader scope. The advantages of internal fixation is safe, don't need more postoperative external fixation, high fusion rate and functional recovery. But the lateral mass plate fixation and pedicle fixation due to anatomical factors make its operation has the certain difficulty and risk. Currently, cervical lateral mass plate screw system is rarely used lock or other lateral mass screws loosen prevention mechanism, so sometimes appear to take off the nail or screw loosening in clinic, especially without auxiliary external fixation after surgery. In 1989, haidt will be its development for titanium alloy implants. Because of titanium in the X-ray and MRI imaging artifacts are less than stainless steel, its application to get a bigger development. Since the biocompatibility of stainless steel and titanium is very good, so the plant without dismantling. Lateral mass around there are many important anatomical structures, the former party with cervical spinal cord, the front for the vertebral artery and the corresponding segment of the nerve root, in order to improve the security of cervical lateral mass plate fixation, avoid screw implant severe nerve, blood vessel damage, and reach the screw through the lateral cortex, lateral double cortex fixation, has many kinds of screw implant of methods, including Roy - Camille, Louis, Magerl, Anderson and AO method, etc. Through the anatomical study, vertebral artery projection in lateral block to the next, nerve root projection direction on the inside to outside, quadrant goes up outside only to be safe. Heller, etc by in vitro experiment found that Roy - Camille method is not easy to damage nerves, but easy to damage small joint surface, and nail short; Advantage is that the operation is easy to grasp, nerve damage rate is low. Magerl method is not easy to damage small joint surface but is in danger of the nerve root injury, nail long; the disadvantage is that operation is difficult, had a higher rate of nerve root injury. These screw implantation methods are put forward on the basis of anatomy, the biggest difficulty lies in the anatomy of the anatomy variation degree is high, so some scholars put forward, in order to increase security, should realize screw implant of personalized, preoperative reference image information know when implanted into the nail position, Angle, screw parameters such as depth, at the same time should improve the design of the steel plate for intraoperative adjust the location of the screws on the steel plate according to the circumstance, in order to achieve the purpose of accurate fixed. 
Cervical segmental vertebral tuberculosis major violation of vertebral body and intervertebral disc, select anterolateral surgical approach to tuberculosis kitchen removal, spinal cord and nerve decompression and bone graft fusion and internal fixation is by far the most common surgical procedure. But for three or more segments of cervical thoracic vertebral tuberculosis, because its span larger lesions area, before, in the column damage is serious, chest and neck segment is concentrated segment of spinal biomechanics, the region after the operation, must want to obtain the strong fixed. If use simple anterior cervical surgery may be caused by internal fixation strength is not enough or bone graft fusion and internal fixation failure. Therefore, choose a combined anterior and posterior approach, not only can fully remove lesions, still can obtain more effective stability reconstruction, in order to reduce postoperative recurrence of tuberculosis, increase the rate of bone graft fusion. But the lack of the operation is to increase the operation time and blood loss and the cost of surgery for patients. Spinal tuberculosis is the main cause of postoperative recurrence of: (1) the destruction of the spinal stability; (2) incomplete lesions cleared; (3) unreasonable postoperative anti-tuberculosis treatment. Cervical thoracic vertebral tuberculosis, although through the issue of posterior surgery can remove the focus thoroughly, and get a good stability, but this study believe that effective measures to prevent the recurrence of tuberculosis, in addition to remove the focus thoroughly and reconstruction of spinal stability, also with the system of anti-tuberculosis treatment have close relations, preoperative line should be effective anti-tuberculosis treatment to prevent intraoperative cause disseminated tuberculosis; Intraoperative anti-tb saline flushing and adopts in the built-in anti-tb drugs lesion area in order to achieve high concentrations of anti-tb drugs and less adverse effect.

At present, the majority of choice in the position of the internal fixation of vertebral and normal vertebral bodies, vertebral surgery need to expose the disease and relatively normal vertebral bodies, with a range of surgical exposure, trauma, for multiple segmental spinal tuberculosis patients, internal fixation with large span, stress concentration, easy to cause the internal fixation loosening and fracture, displacement, due to the fixed range, at the expense of the normal section, postoperative patients with restricted movement, the adjacent segment degeneration accelerated, at the same time a longer piece of bone graft and by stress is big, easy to emerge, subsidence, fracture, etc. Spinal tuberculosis operation in order to eradicate tuberculosis kitchen, Dong Binqu believes such as local expression of spinal tuberculosis is systemic tuberculosis, lesions cleared completely unable to achieve aseptic condition, therefore can only be relative "thoroughly lesions cleared", effective anti-tb drugs and good stability reconstruction is still the main aspects of spinal tuberculosis treatment. Most spinal tuberculosis is more vertebral body destruction, in addition to individual vertebral serious damage, collapse, or into outside the "job" the need for removal of vertebral canal, most of the vertebral tuberculosis only need to thoroughly remove necrotic lesion area, it is not necessary to unlimited expanding scope of surgical resection. As a result, often after lesion area thoroughly remove, disease of vertebral also remain a long segment.

\section{Conclusion}

Therefore, the author thinks that under the precondition of mastering the anatomic relationship there, the surgical approach for cervical thoracic tuberculosis kitchen cleared, bone graft fusion is safe and effective, can be used as C5 T4 vertebral tuberculosis in front of the spinal cord decompression and vertebral resection the preferred approach. One stage anterior internal fixation treatment of cervical vertebra and early cervical segmental spinal can obtain satisfactory curative effect. Thoroughly remove lesions $360^{\circ}$, the treatment that jointed before and after internal fixation and bone graft system of anti-tuberculosis treatment is the key to obtain satisfactory curative effect.

\section{Acknowledgement}

This research was financially supported by the National Science Foundation. 


\section{References}

[1] Rex A. W. Marco MD,Ryan M. Stuckey MD, Stephanie P. Holloway MHA. Prolonged Bed Rest as Adjuvant Therapy After Complex Reconstructive Spine Surgery[J]. Clinical Orthopaedics and Related Research. 2012 (6)

[2] Guohua Lü,Bing Wang,Jing Li, Weidong Liu, Ivan Cheng. Anterior debridement and reconstruction via thoracoscopy-assisted mini-open approach for the treatment of thoracic spinal tuberculosis: minimum 5-year follow-up[J]. European Spine Journal. 2012 (3)

[3] Xiaobing Pu,Qiang Zhou,Qinyi He,Fei Dai, Jianzhong Xu,Zehua Zhang,Kopjar Branko. A posterior versus anterior surgical approach in combination with debridement, interbody autografting and instrumentation for thoracic and lumbar tuberculosis[J]. International Orthopaedics. 2012 (2)

[4] Anil Kumar Jain,Saurabh Jain. Instrumented stabilization in spinal tuberculosis[J]. International Orthopaedics. 2013 (2)

[5] Hongqi Zhang,Shu Huang,Hubing Guo,Lei Ge,Bin Sheng,Yuxiang Wang,Chaofeng Guo,Mingxing Tang. A clinical study of internal fixation, debridement and interbody thoracic fusion to treat thoracic tuberculosis via posterior approach only[J]. International Orthopaedics. 2013(2)

[6] Sudhir Kapoor,Saurabh Kapoor,Mayank Agrawal,Pankaj Aggarwal,Brijesh Kumar Jain. Thoracoscopic decompression in Pott's spine and its long-term follow-up[J]. International Orthopaedics. 2013(2)

[7] S. Rajasekaran,Kamath Vijay,Ajoy Prasad Shetty. Single-stage closing-opening wedge osteotomy of spine to correct severe post-tubercular kyphotic deformities of the spine: a 3-year follow-up of 17 patients[J]. European Spine Journal. 2012(4)

[8] LY Dai,LS Jiang,W Wang.Single-stage anterior autogenous bone grafting and instrumentation in the surgical management of spinal tuberculosis. SPINE. 2012(7)

[9] Zhang HQ, Wang YX, Guo CF, et al.One-stage posterior ap-proach and combined interbody and posterior fusion for thoracolum-bar spinal tuberculosis with kyphosis in children. Orthopedics. 2012(6)

[10] FUKUTA S,MIYAMOTOK,MASUDA T,et al.Two-stage(posterior and anterior)surgical treat ment using posterio spinal instrumentation for pyogenic and tuberculotic spondylitis. SPINE. 2012(6)

[11] EM Halpern,SA Bacon,T Kitagawa,SJ Lewis.Posterior transdiscal three-column shortening in the surgical treatment of vertebral discitis/osteomyelitis with collapse. SPINE. 2013(8) 